\title{
Phenotypic Detection of AmpC B-Lactamases in Gram Negative Bacteria
}

\author{
Fazlul Karim ${ }^{1,2}$, Harunur Rashid ${ }^{1}$, Abu Naser Ibne Sattar ${ }^{1}$, Sharmeen Ahmed ${ }^{1}$, Ahmed Abu Saleh ${ }^{1}$, Humayun Sattar1, Md. Ruhul \\ Amin Miah ${ }^{1}$ \\ 'Department of Microbiology and Immunology, Bangabandu Sheikh Mujib Medical University, Shahbag, Dhaka; ${ }^{2}$ Currently, \\ Department of Pathology, Mymensingh Medical College, Mymensingh
}

\begin{abstract}
This study was carried out to detect AmpC B-lactamase producing Gram-negative bacteria. A total of 374 bacterial isolates from primary cultures of wound $\operatorname{swab}(n=196)$ and urine $(n=178)$ specimens collected from BSMMU and DMCH were investigated. Among the total 374 isolates, 344 (91.98\%) were Gram-negative and $30(8.02 \%)$ were Gram-positive bacteria. Majority of the Gram-negative bacterial isolates were Escherichia coli isolated from 160 (46.52\%) of the cases followed by Pseudomonas species (74, 19.79\%), Klebsiella species (42, 11.23\%), Enterobacter species $(28,7.49 \%)$ and Enterococcus species (20,5.35\%). All isolates were subjected to antimicrobial susceptibility test by Kirby-Bauer disc diffusion method and Gram-negative 1st line-drug-resistant isolates were tested for AmpC ß-lactamase production by disc approximation test. Among 344 Gram-negative bacteria isolated, 183 (53.20\%) were 1st-line-drug-resistant of which AmpC B-lactamase was detected in 51 (27.87\%) isolates. The highest rate of AmpC Blactamase production was observed among Enterobacter spp. (5/10, 50\%), followed by $E$. coli $(23 / 74,31 \%)$, Klebsiella spp. (7/27, 25.92\%), Pseudomonas spp. (11/45, 24.44\%), Acinetobacter spp. (3/15, 20\%) and Proteus spp. (2/12, 16.67\%). Among individual samples, AmpC positive strains were found as the highest in isolates from urine (22/69, $31.88 \%$ ), closely followed by in burn wounds $(30.88 \%)$ and surgical wounds $(\mathbf{1 7 . 3 9 \%})$. AmpC B-lactamase producing $E$. coli was found as the highest in burn wounds (50\%), Klebsiella spp. in urine samples (30.77\%), Pseudomonas spp. in burn and surgical wounds (25\%), Enterobacter spp. in burn wounds (50\%), and Acinetobacter spp. in urine (28.57\%). Proteus spp. was found only in burn wounds (22.22\%). All AmpC B-lactamase producers were sensitive to Imipenem (100\%). Considerable numbers of AmpC producing bacteria were detected from wound infection and urinary tract infection cases which indicate that $\mathrm{AmpC}$ is a major threat for antibiotic therapy.
\end{abstract}

Key words: AmpC B-lactamase, Gram-negative bacteria, Wound Swab, Urine

\section{Introduction}

The most common mechanism of resistance to ß-lactam antibiotics in Gram-negative bacilli is the expression of $\beta$ -

Correspondence:

Dr. Fazlul Karim

Lecturer, Department of Pathology,

Mymensingh Medical College, Mymensingh lactamases, which are found responsible for majority of the therapeutic failures. In addition, chromosomal ß-lactamases seem to be associated more frequently than before with bacterial resistance. ${ }^{1}$ The introduction of third-generation cephalosporins has been an important step in the successful treatment of Enterobacter, Serratia and Pseudomonas species. ${ }^{2}$ There are several reports of broad spectrum $\beta$-lactam 
resistance among bacteria emerging during therapy with a single $\beta$-lactam antibiotic. ${ }^{3}$ The mechanism of this resistance was found due to mutations leading to derepression of an inducible chromosomally determined cephalosporinase. ${ }^{4,5}$

AmpC ß-lactamases refers to a family of related enzymes, produced in a variety of members of the family Enterobacteriacae. Over 20 years ago, an Ampicillin-resistant mutant was isolated that produced elevated levels of chromosomal ß-lactamase. The mutant was denoted AmpA and was shown to affect a regulatory region for the $\beta$ lactamase gene. ${ }^{6}$

AmpC B-lactamases: (1) are Cephalosporinases not usually inhibited by Clavulanic acid; (2) hydrolyze Cephamycins as well as other extended-spectrum Cephaloporins; (3) are usually chromosomally mediated (may also be plasmid mediated); (4) commonly found in Pseudomonas species, Klebsiella pneumoniae, Enterobacter species, Acinetobacter species, E. coli and Citrobacter species etc.

The majority of $\beta$-lactamases of Gram-negative bacteria is expressed constitutively. However in 1967, Hennessey described an inducible B-lactamase of Enterobacter cloacae that is known to be encoded by the chromosomal $A m p C$ gene. ${ }^{7}$

Studies on detection of AmpC production are much fewer than Extended Spectrum Beta-lactamase (ESBL) detection. In Australia, a study showed that AmpC was induced in $68 \%$ of the 326 organisms found capable of $\beta$-lactamase induction. ${ }^{8}$ The European study group on antibiotic resistance in 1987 showed that the strains with inducible $\beta$-lactamases were $11 \%$ of all Gram-negative bacteria. In a study in India, among 318 routine isolates of enteric bacilli, 89 isolates were found susceptible to Expanded Spectrum Cephalosporins (ESC) by Kirby-Bauer disc diffusion test. However, when they were tested by disc approximation method, 56 were found to be resistant to ESC.9,10 Another study in India showed that out of 286 isolates, 151 were ESBL-producers of which 131 (46\%) were also derepressed mutants, 40 (14\%) being plainly derepressed. ${ }^{11}$ Inducible AmpC B-lactamase production was detected in $19(7 \%)$ of the isolates in the study. No National Committee for Clinical Laboratory Standards (NCCLS), USA recommendation exists for detection of AmpC ß-lactamases. ${ }^{12}$

Several AmpC detection tests have been proposed, based on clinical Microbiology techniques are: Screening for AmpC ßlactamases producing bacteria, Modified three dimensional tests Disc approximation method, Cefoxitin agar media (CAM) based assay, Extraction of B-lactamase, and Analysis by isoelectric focusing, Detection and Characterization of $B$ lactamases by PCR amplification and DNA sequencing, Detection of $A m p R$ regulatory gene, and Pulsed-field Electrophoresis. Till now there is no recognized gold standard test for detection of AmpC B-lactamases.

Disc approximation test is a simple test, easy to perform, low cost, can be easily performed and can easily be incorporated into the routine clinical laboratory practices for detection of $A m p C$ gene. ${ }^{8}$

It is highly desirable that clinical laboratories should undertake such additional tests capable of detecting latent resistance intime so that they can effectively guide therapeutic as well as infection control strategies. The present study of phenotypic detection of AmpC B-lactamase producing bacteria will prevent unnecessary use of antibiotics and benefit patients by administration of appropriate antibiotics. This will also reduce number of hospital stay and reduce treatment cost of the patient and thus help in reducing national health expenditure.

\section{Methods}

A total of 374 samples consisting of wound swab from burn and surgical wounds and urine were collected from patients of indoor and outpatient departments (OPD) of Bangabandu Sheikh Mujib Medical University (BSMMU) and Dhaka Medical College (DMC) Hospitals.

Specimens of wound swabs were collected from Burn unit, Surgery units, Casualty units, Orthopaedic units and Surgery OPD of DMCH and BSMMU hospital and urine samples were collected only from OPD of BSMMU.

All wound swabs were inoculated on Blood agar media and MacConkey's agar media and incubated at $37^{\circ} \mathrm{C}$ for $16-18$ 
hours. After proper incubation, suspected colonies were identified by colony morphology, staining character, coagulase test, catalase test, haemolytic property and other biochemical tests.

All bacterial isolates were subjected to antibiotic sensitivity test by Kirby-Bauer disc diffusion method as described previously.

Isolated Gram-negative bacteria showing 1st-line-drug resistance were further studied by the disc approximation test to detect AmpC ß-lactamase production.

Disc approximation test procedure: ${ }^{9}$

In this procedure, antimicrobial susceptibility was tested for two third generation Cephalosporims (Cefoxitin and Cefotaxime). For this purpose, 3-5 colonies of the test organism were picked up from the pure culture plate, suspended in $5 \mathrm{ml}$ of sterile normal saline and the turbidity produced was then adjusted to that of $0.5 \mathrm{McF}$ arland standard tube no. 1. Within 15 minutes, a sterile cotton wool swab was dipped into the bacterial suspension and then streaked on a dried surface of Muller-Hinton plate to get a uniform distribution of the inoculum. Then a Cefoxitin disc (after becoming equivalent to room temperature) was placed on the surface of inoculated plate. A Cefotaxime disc was placed again at $2.5 \mathrm{~cm}$ (centre to centre) from the Cefoxitin disc. The plate was then incubated at $37^{\circ} \mathrm{C}$ for $16-18$ hours and reading was taken on the next day. AmpC B-lactamase production was positive if there was a flattening of zone of inhibition of Cefotaxime towards Cefoxitin by $\geq 4 \mathrm{~mm}$. This indicated that the organism was inducible ß-lactamase producer. When there was resistance in both Cefotaxime and Cefoxitin, the organism was considered as derepressed mutant.

\section{Results}

A total of 478 specimens consisting of 140 burn wounds, 160 sergical wound swabs and 178 urine were investigated from which 374 (78.24\%) bacteria were isolated. Among them, $113(80.71 \%)$ bacterial isolates were from burn wounds, 83 (51.87\%) from surgical wounds, and 178 (100.0\%) from urine. (Table I)
Out of 374 isolates, 344 (91.98\%) were Gram-negative and $30(8.02 \%)$ were Gram-positive bacteria. Among the Gramnegative bacteria, majority were E. coli 160 (46.51\%), followed by Pseudomonas (74, 21.51\%), Klebsiella (42, $12.21 \%)$, Enterobacter $(28,8.14 \%)$, and Acinetobacter (22, $6.40 \%)$. Out of total 344 Gram-negative bacteria, 182 (52.91\%) were isolated from wound and 162 (47.09\%) from urine samples. (Table II)

Table I. Rate of isolation of bacteria from study population $(n=478)$.

\begin{tabular}{lll}
\hline Types of sample & Number of samples & No. of isolated bacteria \\
\hline Burn wound & 140 & $113(80.71)$ \\
Surgical wound & 160 & $83(51.87)$ \\
Urine & 178 & $178(100.0)$ \\
Total & 478 & $374(78.24)$ \\
\hline
\end{tabular}

Figures in parentheses indicate percentages

Table II. Distribution of Gram-negative bacterial species isolated from various samples $(n=344)$

\begin{tabular}{|c|c|c|c|c|}
\hline $\begin{array}{l}\text { Bacterial species } \\
\text { isolated }\end{array}$ & $\begin{array}{l}\text { No. of } t \\
\text { Burn wound } \\
(n=103)\end{array}$ & $\begin{array}{l}\text { bacterial isolates } \mathrm{f} \\
\text { Surgical wound } \\
(\mathrm{n}=79)\end{array}$ & $\begin{array}{l}\text { from- } \\
\text { Urine } \\
(\mathrm{n}=162)\end{array}$ & $\begin{array}{l}\text { Total Gram- } \\
\text { negative bacteria } \\
(\mathrm{n}=344)\end{array}$ \\
\hline E.coli & $31(30.10)$ & $34(43.04)$ & $95(58.64)$ & $160(46.51)$ \\
\hline Klebsiella & $12(11.65)$ & $10(12.66)$ & $20(12.35)$ & $42(12.21)$ \\
\hline Proteus & $10(9.71)$ & $5(6.33)$ & $3(1.85)$ & $18(5.23)$ \\
\hline Pseudomonas & $35(33.98)$ & $21(36.58)$ & $18(11.11)$ & $74(21.51)$ \\
\hline Enterobacter & $8(7.76)$ & $4(5.06)$ & $16(9.88)$ & $28(8.14)$ \\
\hline Acinetobacter & $7(6.80)$ & $5(6.33)$ & $10(6.17)$ & $22(6.40)$ \\
\hline
\end{tabular}

Some 183 of the Gram-negative bacteria were 1st-line-drug resistant of which 51 (27.87\%) were AmpC positive. Rate of AmpC positivity were found the highest among isolates from urine where out of 69 strains, 21 (31.88\%) were AmpC positive. On the other hand in surgical wound samples, out of 46 strains, 8 (17.39\%) were AmpC positive. In burn wound 
samples, out of 68 strains, 17 (30.88\%) were AmpC positive. (Table III)

Table III. Results of AmpC B-lactamases among the 1st-linedrug-resistant Gram-negative bacteria in different type of samples

\begin{tabular}{lll}
\hline Type of sample & $\begin{array}{l}\text { No. of 1st line drug } \\
\text { resistant strains }\end{array}$ & $\begin{array}{l}\text { No. of AmpC B-lactamase } \\
\text { positive strains }\end{array}$ \\
\hline Burn wound & 68 & $21(30.88 \%)$ \\
Surgical wound & 46 & $8(17.39 \%)$ \\
Urine & 69 & $22(31.88 \%)$ \\
Total & 183 & $51(27.87 \%)$ \\
\hline
\end{tabular}

Out of 183 1st-line-drug-resistant Gram-negative bacteria, AmpC producing organism was 51 (27.87\%). Considering AmpC positivity rate, it was found the highest in Enterobacter species (5/10, 50\%), followed by E. coli $(23 / 74$, $31.08 \%$ ), Klebsiella species $(7 / 27,25.92 \%)$, Pseudomons species $(11 / 45,24.44 \%)$, Acinetobacter species $(3 / 15,20 \%)$, and Proteus species $(2 / 12,16.67 \%)$. (Table IV)

Table IV. Rate of AmpC positivity among the strains tested $(n=183)$.

\begin{tabular}{lll}
\hline Name of strain & No. of strains tested & $\begin{array}{l}\text { No. of AmpC B - } \\
\text { lactamae }\end{array}$ \\
\hline E. coli & 74 & $23(31.08)$ \\
Klebsiella spp. & 27 & $7(25.92)$ \\
Proteus spp. & 12 & $2(16.67)$ \\
Pseudomonas spp. & 45 & $11(24.44)$ \\
Enterobacter spp. & 10 & $5(50.0)$ \\
Acinetobacter spp. & 15 & $3(20.0)$ \\
Total & 183 & $51(27.87)$ \\
\hline
\end{tabular}

Figures in parentheses indicate percentages

\section{Discussion}

Bacterial antibiotic resistance has become a major clinical concern worldwide including Bangladesh. ${ }^{12}$ Failure to detect the enzymes like Extended Spectrum $\beta$-lactamases (ESBLs), AmpC B-lactamases, Metallo ß-lactamases has contributed to their uncontrolled spread and therapeutic failures. ${ }^{11}$
In this study, phenotypic detection of AmpC $\beta$-lactamases among Gram-negative bacterial isolates was done. No AmpC producer Proteus species was found among isolates from surgical wounds and urine specimens, and no AmpC producer Acinetobacter species was isolated from surgical wounds as well. This phenomenon may be due to less number of Proteus and Acinetobacter isolated from the samples.

Though there were many limitations, this study was conducted for the first time in Bangladesh revealing the extent of AmpC producing organisms responsible for various infections in two large hospitals in Dhaka City. Antimicrobial sensitivity tests being used now are unable to detect AmpC B-lactamase mediated drug resistance against 3rd generation Cephalosporins which may lead to treatment failure.

Antibiotics including 3rd generation Cephalosporins are being indiscriminately and irrationally used so that there is increasing number of occurrences of antibiotic resistance among Gramnegative bacteria. It may be concluded from the present study that there are considerable number of AmpC $\beta$-lactamase producing bacteria responsible for sepsis and urinary tract infections. AmpC B-lactamases cause problem in the treatment of any infection caused by as such organisms which are resistant by nature to most antibiotics.

From the present study the following recommendations can be made: (1) Phenotypic detection method should be incorporated in routine susceptibility tests; (2) Irrational use of antibiotics especially 3rd generation Cephalosporins should be prohibited; and (3) In case of AmpC positive strains, all Cephalosporins including 3rd generation should be avoided and the treatment of choice would be Imipenem or Meropenem.

\section{References}

1. Bush K. Recent developments in B-lactamase research and their implications for the future. Rev Infect Dis 1988; 10(4): 681- 690.

2. Gutmann L, Williamson R. A model system to demonstrate that ß-lactamase-associated antibiotic trapping could be a potential means of resistance. J Infect Dis 1983; 148(2): 316-321.

3. Olson B, Weinstein RA, Nathan C, Kabins SA. Broad-spectrum ß-lactrum resistance in Enterobacter: emergence during treatment and mechanisms of resistance. J Antimicrob Chemother 1983; 11: 299-310.

4. Lampe MF, Allaie BJ, Minshow BH, Sherris JC. Mutational 
enzymatic resistance of Enterobacter species to Beta-lactam antibiotics. Antimicrob Agents Chemother 1982; 21(4): 655660 .

5. Gootz TD, Sanders CC, Goerin RV. Resistance to Cefamandole: Derepression of $\beta$-lactamases by Cefotixin and mutation in Enterobacter cloacae. J Infect Dis 1982; 140 (1): 34-42.

6. Lindberg F, Normark S. Contribution of chromosomal Blactamase to $ß$-lactam resistance in Enterobacteria. Rev Infect Dis 1986; 8 (Suppl. 3): S292-S303.

7. Henessey TD. Inducible ß-lactamases in Enterobacter. J Gen Microbiol 1967; 49: 277-285.

8. Moritz VA, Carson PBP. Cefoxitin sensitivity as a marker for inducible beta-lactamases. J Med Microbiol 1986; 21: 203-207.

9. Sanders CC, Sanders WE. Emergence of resistance to Cefamandol: possible role of Cefoxitin-inducible Beta- lactamases. Antimicrob Agents Chemother 1979; 15(6): 792797.

10. Revathi G, Singh S. Detection of extended-spectrum Cephalosporin resistance due to inducible lactamases in hospital isolates. Indian J Med Microbiol 1997; 15(3): 113-115.

11. Rodrigues C, Johi P, Jani SH, Alphonse M, Radhakrishnan R, Meheta A. Detection of ß-lactamases in nosocomial Gramnegative clinical isolates. Indian J Med Microbiol 2004; 22 (4): 247-250.

12. Thomson KS. Controversies about extended-spectrum and AmpC ß-lactamases. Emerg Infect Dis 2001; 7(2): 333- 336.

13. Karim M, Ahmed S, Parvez M. Emerging multi-drug resistant organim in tertiary care hospital of Dhaka city. Bangladesh $\mathrm{J}$ Med Sc 2002; $8: 53-57$. 